\title{
A personal tour through symplectic topology and geometry
}

\section{Miguel Abreu}

Departamento de Matemática, Instituto Superior Técnico, Av. Rovisco Pais, 1049-001 Lisboa, Portugal

E-mail address: mabreu@math.ist.utl.pt

\section{Introduction}

In this survey I will present a very personal tour through symplectic topology and geometry, describing the following three paths and the way most of my work fits in them.

(i) Gromov's Compactness Theorem for pseudo-holomorphic curves in symplectic manifolds ([23]) and the topology of symplectomorphism groups of rational ruled surfaces (sections 2 and 3, references [1, 2]).

(ii) Atiyah-Guillemin-Sternberg's Convexity Theorem for the moment map of Hamiltonian torus actions $([9,25])$ and Kähler geometry of toric orbifolds in symplectic coordinates (sections 4 and 5, references $[3,4,5])$.

(iii) Donaldson's moment map framework for the action of the symplectomorphism group on the space of compatible almost complex structures ([17]) and the topology of the space of compatible integrable complex structures of a rational ruled surface (sections 6 and 7 , references $[6,7])$.

Key words: pseudo-holomorphic curves, symplectomorphism groups, moment maps, extremal metrics, compatible complex structures.

Miguel Abreu is supported in part by FCT through program POCTI-Research Units Pluriannual Funding Program and grant POCTI/MAT/57888/2004.

Acknowledgments: I would like to thank my advisor, Yakov Eliashberg, and the collaborators I had in the work described in this survey: Dusa McDuff, Gustavo Granja and Nitu Kitchloo. My mathematical life has been much easier because of them. 


\section{Pseudo-holomorphic Curves in Symplectic Manifolds}

In this section we define compatible almost complex structures on symplectic manifolds and discuss Gromov's Compactness Theorem for their pseudo-holomorphic curves. We will also present two special geometric properties that pseudo-holomorphic curves have in dimension 4. For a further understanding of pseudo-holomorphic curves and its applications to symplectic topology see [29], where you can also find a detailed list of references.

Almost complex manifolds. Recall that an almost complex manifold is a pair $(M, J)$, where $M$ is a smooth manifold and $J: T M \rightarrow T M$ is an endomorphism of its tangent bundle such that

$$
J_{p}^{2}=-\mathrm{id}_{p}: T_{p} M \rightarrow T_{p} M, \forall p \in M .
$$

The condition $J^{2}=-$ id implies that the dimension of any almost complex manifold is even.

Any complex manifold is an almost complex manifold with an integrable almost complex structure. In real dimension 2 , any almost complex manifold is a complex curve, i.e. a Riemann surface $(\Sigma, j)$.

\section{Pseudo-holomorphic curves.}

Definition 2.1. A parametrized pseudo-holomorphic curve is a map from a Riemann surface to an almost complex manifold,

$$
f:(\Sigma, j) \rightarrow(M, J)
$$

such that

$$
d f \circ j=J \circ d f .
$$

Its image $C=f(\Sigma)$ is an unparametrized pseudo-holomorphic curve. We might also write $J$-holomorphic curve, instead of pseudo-holomorphic curve.

Remark 2.2.

(i) The Cauchy-Riemann equation (1) gives rise to a quasi-linear first order elliptic system of PDE's with good analytical properties: smooth solutions, removal of singularities, etc.

(ii) Any immersed real 2-dimensional surface $C \rightarrow(M, J)$ with complex tangent space, i.e. such that $J(T C)=T C$, is an unparametrized pseudo-holomorphic curve. For example, any complex curve in a complex manifold. 


\section{Compatible almost complex structures.}

Definition 2.3. A compatible almost complex structure on a symplectic manifold $(M, \omega)$ is an almost complex structure $J$ on $M$ such that

$$
\langle\cdot, \cdot\rangle_{J} \equiv \omega(\cdot, J \cdot)
$$

is a Riemannian metric on $M$. This is equivalent to $\omega(J \cdot, J \cdot)=\omega(\cdot, \cdot)$ and $\omega(X, J X)>0, \forall 0 \neq X \in T M$.

The space of all compatible almost complex structures on a symplectic manifold $(M, \omega)$ will be denoted by $\mathcal{J}(M, \omega)$.

\section{Remark 2.4.}

(i) $\mathcal{J}(M, \omega)$ is non-empty, infinite-dimensional and contractible, for any symplectic manifold $(M, \omega)$. In particular, any $(M, \omega)$ has well defined Chern classes $c_{k} \equiv c_{k}(M, \omega) \in H^{2 k}(M, \mathbb{Z})$.

(ii) A Kähler manifold is a symplectic manifold $(M, \omega)$ with an integrable compatible complex structure $J$.

Gromov's Compactness Theorem. Let $(M, \omega)$ be a symplectic manifold and $J \in \mathcal{J}(M, \omega)$ a compatible almost complex structure. We will now illustrate how the compatibility condition provides enough apriori geometric control on pseudo-holomorphic curves for a satisfactory global theory to hold.

Fix an homology class $A \in H_{2}(M, \mathbb{Z})$ and let $f:(\Sigma, j) \rightarrow(M, J)$ be a closed pseudo-holomorphic curve whose image $C=f(\Sigma) \subset M$ represents $A$, i.e. such that

$$
[C]=f_{*}([\Sigma])=[A] \in H_{2}(M, \mathbb{Z}) .
$$

Proposition 2.5. The area of $C$ with respect to $\langle\cdot, \cdot\rangle_{J} \equiv \omega(\cdot, J \cdot)$ is given by

$$
\operatorname{area}(C)=\int_{C} \omega=[\omega](A),
$$

and is minimal within the class of submanifolds of $M$ representing the homology class $A$.

Proof. This result follows from the following linear algebra inequality, known as Wirtinger's inequality:

$$
\omega(X, Y)=\langle J X, Y\rangle \leq\|J X\|\|Y\|=\|X\|\|Y\|,
$$

with equality iff $Y$ is a multiple of $J X$.

Hence, on a symplectic manifold with a compatible almost complex structure,

homology controls the area of pseudo-holomorphic curves. 
In 1985, Gromov [23] realized that this fact could be used to prove a compactness theorem for pseudo-holomorphic curves. The following is a simple version of that theorem, that is however enough for the applications we will discuss in this lecture.

Theorem 2.6. [Gromov] Let $(M, \omega)$ be a compact symplectic manifold and

$$
f_{n}:(\Sigma, j) \rightarrow\left(M, J_{n}\right)
$$

a sequence of pseudo-holomorphic curves representing a fixed homology class $0 \neq A \in H_{2}(M, \mathbb{Z})$. Assume that $\Sigma$ is closed, $J_{n} \in \mathcal{J}(M, \omega)$ and there exists a $J_{\infty} \in \mathcal{J}(M, \omega)$ such that $J_{n} \rightarrow J_{\infty}$ smoothly. Then,

(i) either there exists a subsequence of $f_{n}$ that smoothly converges to a pseudo-holomorphic curve

$$
f_{\infty}:(\Sigma, j) \rightarrow\left(M, J_{\infty}\right)
$$

also representing the homology class $A$ (if $\Sigma$ contains spherical components, the non-compact reparametrization group $S L(2, \mathbb{C})$ needs to be taken into account here);

(ii) or there exists a $J_{\infty}$-holomorphic spherical "bubble", i.e. the class $A$ can be written as

$$
A=B+A^{\prime} \in H_{2}(M, \mathbb{Z}),
$$

where $0 \neq B \in H_{2}(M, \mathbb{Z})$ can be represented by a $J_{\infty}$-holomorphic sphere (the "bubble") and $0 \neq A^{\prime} \in H_{2}(M, \mathbb{Z})$ can be represented by a $J_{\infty}$-holomorphic curve.

The positivity in the compatibility condition between $J$ and $\omega$ implies that a necessary condition for a homology class $A \in H_{2}(M, \mathbb{Z})$ to be represented by a $J$-holomorphic curve is

$$
[\omega](A)>0 .
$$

This simple fact implies the following corollary to Theorem 2.6.

Corollary 2.7. If the homology class $A \in H_{2}(M, \mathbb{Z})$ cannot be written as $A=A_{1}+A_{2}$, where $[\omega]\left(A_{i}\right)>0, i=1,2$, and either $A_{1}$ or $A_{2}$ is a spherical class, then (i) in Theorem 2.6 holds and no "bubbles" can occur.

Properties of pseudo-holomorphic curves in dimension 4. It turns out that in dimension four homology controls important geometric properties of pseudo-holomorphic curves. These will be used later on.

Theorem 2.8. [Positivity of Intersections] Two distinct closed $J$-holomorphic curves $C$ and $C^{\prime}$ in an almost complex 4-manifold $(M, J)$ have only a finite number of intersection points. Each such point $x \in$ $C \cap C^{\prime}$ contributes a number $k_{x} \geq 1$ to the algebraic intersection number 
$[C] \cdot\left[C^{\prime}\right] \in \mathbb{Z}$. Moreover, $k_{x}=1$ if and only if the curves $C$ and $C^{\prime}$ intersect transversally at $x$.

\section{Remark 2.9.}

(i) This Theorem implies that two J-holomorphic curves always intersect positively. This is not true for intersections of symplectic submanifolds. For example, one can easily find two symplectic planes in $\left(\mathbb{R}^{4}, \omega_{\mathrm{st}}\right)$ with a negative transversal intersection at the origin.

(ii) This Theorem also implies that if $C$ and $C^{\prime}$ are distinct $J$-holomorphic curves then

$$
[C] \cdot\left[C^{\prime}\right]=0 \Rightarrow C \text { and } C^{\prime} \text { are disjoint. }
$$

Let $f:(\Sigma, j) \rightarrow\left(M^{4}, J\right)$ be a pseudo-holomorphic curve, $g \equiv$ genus of $\Sigma$ and $C=f(\Sigma) \subset M$. Assume that $f$ is somewhere injective, i.e.

there exists $z \in \Sigma$ such that $d f(z) \neq 0$ and $f^{-1}(f(z))=\{z\}$.

This condition avoids the trivial constant pseudo-holomorphic curve and multiple coverings of a fixed pseudo-holomorphic map.

Definition 2.10. The virtual genus of $C$ is defined to be the number

$$
g(C)=1+\frac{1}{2}\left([C] \cdot[C]-c_{1}([C])\right)
$$

where $c_{1} \in H^{2}(M, \mathbb{Z})$ is the first Chern class of the complex vector bundle $(T M, J)$ over $M$.

Theorem 2.11. [Adjunction Formula] The virtual genus $g(C)$ is an integer. Moreover,

$$
g(C) \geq g
$$

with equality iff $C$ is embedded.

\section{The Symplectomorphism Groups of $S^{2} \times S^{2}$}

In this section we show how pseudo-holomorphic curves can be used to study the topology of the symplectomorphism groups of $S^{2} \times S^{2}$. There is an analogous story for the nontrivial $S^{2}$-bundle over $S^{2}$ (see [2] and [7]).

Pseudo-holomorphic spheres in $S^{2} \times S^{2}$. Consider the symplectic manifold $\left(S^{2} \times S^{2}, \omega=\sigma \times \sigma\right)$, where $\sigma$ denotes the standard area form on $S^{2}$ with $\int_{S^{2}} \sigma=1$. Let $A$ and $B$ denote the homology classes

$$
A=\left[S^{2} \times\{p\}\right] \text { and } B=\left[\{p\} \times S^{2}\right] \quad \text { in } H_{2}\left(S^{2} \times S^{2}, \mathbb{Z}\right) .
$$


Proposition 3.1. For any compatible almost complex structure $J \in \mathcal{J}\left(S^{2} \times\right.$ $\left.S^{2}, \omega\right)$ and any point $p \in S^{2} \times S^{2}$, there exist a J-holomorphic sphere representing the homology class $A$ and a J-holomorphic sphere representing the homology class $B$, both passing through the point $p$.

Proof. The subset of $\mathcal{J}\left(S^{2} \times S^{2}, \omega\right)$ formed by compatible almost complex structures for which the statement of the proposition is true is:

(i) non-empty, since it contains the standard split complex structure $J_{0}=j_{0} \times j_{0}$

(ii) open, because the pseudo-holomorphic equation (1) is elliptic;

(iii) closed, by Gromov's compactness Theorem 2.6 and Corollary 2.7.

If we consider on $S^{2} \times S^{2}$ the split symplectic form

$$
\omega_{\lambda}=\lambda \sigma \times \sigma \quad \text { with } \quad 1<\lambda \in \mathbb{R},
$$

the homology class $A$ no longer satisfies the conditions of Corollary 2.7 (although the smaller class $B$ still does). In fact, $A$ can be written as

$$
A=(A-B)+B, \text { with }\left[\omega_{\lambda}\right](A-B)=\lambda-1>0 \text { and }\left[\omega_{\lambda}\right](B)=1>0 .
$$

Moreover, the anti-diagonal

$$
\bar{D}=\left\{(p,-p): p \in S^{2}\right\} \subset S^{2} \times S^{2}
$$

is an embedded symplectic sphere representing the homology class $(A-B)$ and one can construct compatible almost complex structures $J \in \mathcal{J}\left(S^{2} \times\right.$ $\left.S^{2}, \omega_{\lambda}\right)$ that make it $J$-holomorphic. Hence, there are compatible almost complex structures $J \in \mathcal{J}\left(S^{2} \times S^{2}, \omega_{\lambda}\right)$ for which the homology class $(A-$ $B$ ) is represented by a $J$-holomorphic sphere. Note that by positivity of intersections, Theorem 2.8, and since

$$
(A-B) \cdot A=A \cdot A-B \cdot A=0-1=-1<0,
$$

whenever there is a $J$-holomorphic sphere representing the class $(A-B)$ there is no $J$-holomorphic sphere representing the class $A$. This means that Proposition 3.1 does not hold as stated for the symplectic manifold $\left(S^{2} \times S^{2}, \omega_{\lambda}=\lambda \sigma \times \sigma\right), 1<\lambda \in \mathbb{R}$, and the homology class $A$ (although it does hold for the smaller class $B$ ).

As we will see, this change in the structure of pseudo-holomorphic spheres, that arises from a variation of the symplectic form, is related to a change in the topology of the corresponding symplectomorphism groups. 
Gromov's Theorem. Let $G$ denote the subgroup of the symplectomorphism group Diff $\left(S^{2} \times S^{2}, \omega=\sigma \times \sigma\right)$ that consists of symplectomorphisms that act as the identity in $H_{2}\left(S^{2} \times S^{2}, \mathbb{Z}\right)$. Note that the full symplectomorphism group $\operatorname{Diff}\left(S^{2} \times S^{2}, \omega\right)$ is a simple $\mathbb{Z}_{2}$-extension of $G$.

Theorem 3.2. (Gromov [23]) $G$ is homotopy equivalent to its subgroup of standard isometries of $S^{2} \times S^{2}$, i.e.

$$
G \sim S O(3) \times S O(3) .
$$

Proof. To simplify notation, denote by $\mathcal{J}$ the contractible space of compatible almost complex structures $\mathcal{J}\left(S^{2} \times S^{2}, \omega\right)$. $G$ acts on $\mathcal{J}$ by conjugation, with isotropy at $J_{0}=j_{0} \times j_{0}$ given by $S O(3) \times S O(3)$. This action is clearly nontransitive since, for example, one cannot send an integrable compatible almost complex structure to a non-integrable one. Hence, $G / S O(3) \times S O(3) \neq \mathcal{J}$.

However, this natural action of $G$ on $\mathcal{J}$ gives us a map

$$
\begin{aligned}
\beta: G / S O(3) \times S O(3) & \rightarrow \mathcal{J} \\
{[\varphi] } & \mapsto \varphi_{*}\left(J_{0}\right),
\end{aligned}
$$

which is still an homotopy equivalence. To prove that, one uses pseudoholomorphic spheres to construct an homotopy inverse

$$
\begin{aligned}
\alpha: \mathcal{J} & \rightarrow G / S O(3) \times S O(3) \\
J & \mapsto\left[\varphi_{J}\right]
\end{aligned}
$$

in the following way.

It follows from Proposition 3.1 that, for any $J \in \mathcal{J}$, there exist two foliations $\mathcal{F}_{J}^{A}$ and $\mathcal{F}_{J}^{B}$ of $S^{2} \times S^{2}$ whose leaves are $J$-holomorphic spheres representing the homology classes $A=\left[S^{2} \times\{p\}\right]$ and $B=\left[\{p\} \times S^{2}\right]$. Note that

(i) the positivity of intersections Theorem 2.8 implies that any two spheres in the same foliation are disjoint, since $A \cdot A=0=B \cdot B$;

(ii) positivity of intersections also implies that each sphere in $\mathcal{F}_{J}^{A}$ intersects each sphere in $\mathcal{F}_{J}^{B}$ at exactly one point and in a transverse way, since $A \cdot B=1$;

(iii) the adjunction formula of Theorem 2.11 implies that all spheres in both foliations are embedded, since in this case

$$
\text { virtual genus }=1+\frac{1}{2}(0-2)=0=\text { genus of } S^{2} .
$$

(iv) the leaves of $\mathcal{F}_{J_{0}}^{A}$ and $\mathcal{F}_{J_{0}}^{B}$ are exactly the spheres $S^{2} \times\{p\}$ and $\{p\} \times S^{2}$, with $p \in S^{2}$. 
Hence, given any $J \in \mathcal{J}$ we can construct a diffeomorphism

$$
\psi_{J}: S^{2} \times S^{2} \rightarrow S^{2} \times S^{2}
$$

that maps the $J_{0}$-foliations to the corresponding $J$-foliations. One checks that, for a diffeomorphism $\psi_{J}$ with this property, the symplectic form $\omega_{J} \equiv$ $\psi_{J}^{*}(\omega)$ is linearly isotopic to $\omega$, i.e.

$$
\omega_{t}=t \omega_{J}+(1-t) \omega, t \in[0,1], \text { is an isotopy of symplectic forms. }
$$

Moser's method can then be used to deform $\psi_{J}$ to a symplectomorphism $\varphi_{J} \in G$.

With appropriate care, this whole construction can be made canonical modulo $S O(3) \times S O(3)$ and the map $\alpha: \mathcal{J} \rightarrow G / S O(3) \times S O(3)$ obtained this way can be checked to be indeed an homotopy inverse to the map $\beta$.

Topology of $G_{\lambda}$. As was already explained, the structure of pseudoholomorphic spheres on $\left(S^{2} \times S^{2}, \omega_{\lambda}=\lambda \sigma \times \sigma\right)$ changes as $\lambda \in \mathbb{R}$ increases from $\lambda=1$ to any value $\lambda>1$. In fact, this structure changes every time $\lambda$ increases past a positive integer value. These changes and the way they relate to the symplectomorphism groups

$$
G_{\lambda} \equiv \operatorname{Diff}\left(S^{2} \times S^{2}, \omega=\lambda \sigma \times \sigma\right)
$$

can be summarized in the following way (see [1], [2] and [28] for further details).

Given $1<\lambda \in \mathbb{R}$, let $\mathcal{J}_{\lambda}$ denote the contractible space of all almost complex structures on $S^{2} \times S^{2}$ compatible with $\omega_{\lambda}$ and $\ell \in \mathbb{N}$ be the unique positive integer such that $\ell<\lambda \leq \ell+1$. Then, there is a stratification $\mathcal{J}_{\lambda}$ of the form

where:

$$
\mathcal{J}_{\lambda}=U_{0} \sqcup U_{1} \sqcup \cdots \sqcup U_{\ell},
$$

(i)

$$
U_{k} \equiv\left\{J \in \mathcal{J}_{\lambda}:(A-k B) \in H_{2}\left(S^{2} \times S^{2} ; \mathbb{Z}\right),\right.
$$

is represented by a $J$-holomorphic sphere\}.

Note that $\left[\omega_{\lambda}\right](A-k B)>0 \Leftrightarrow k \leq \ell$.

(ii) $U_{0}$ is open and dense in $\mathcal{J}_{\lambda}$. For $k \geq 1, U_{k}$ has codimension $4 k-2$ in $\mathcal{J}_{\lambda}$.

(iii) $\overline{U_{k}}=U_{k} \sqcup U_{k+1} \sqcup \cdots \sqcup U_{\ell}$.

(iv) Each stratum $U_{k}$ has an integrable element $J_{k} \in U_{k}$ (such that $\left(S^{2} \times S^{2}, J_{k}\right) \cong(2 k)$-Hirzebruch surface, see section 7$)$, for which the Kähler isometry group

$$
K_{k} \equiv \operatorname{Isom}\left(S^{2} \times S^{2},\langle\cdot, \cdot\rangle_{\lambda, k} \equiv \omega_{\lambda}\left(\cdot, J_{k} \cdot\right)\right)
$$


is such that

$$
K_{k} \cong \begin{cases}S O(3) \times S O(3), & \text { if } k=0 \\ S^{1} \times S O(3), & \text { if } k \geq 1\end{cases}
$$

(v) The inclusion

$$
\begin{aligned}
\left(G_{\lambda} / K_{k}\right) & \longrightarrow U_{k} \\
{[\varphi] } & \longmapsto \varphi_{*}\left(J_{k}\right)
\end{aligned}
$$

is a weak homotopy equivalence.

Although we do not know apriori the topology of the strata $U_{k}$, the fact that each is homotopy equivalent to a quotient of $G_{\lambda}$ and that their union is the contractible space $\mathcal{J}_{\lambda}$ can be used to obtain important information regarding the topology of $G_{\lambda}$, in particular its rational cohomology ring.

Theorem 3.3. [2] When $\lambda>1$,

$$
H^{*}\left(G_{\lambda} ; \mathbb{Q}\right)=\Lambda(a, x, y) \otimes S\left(w_{\ell}\right),
$$

where $\Lambda(a, x, y)$ denotes the exterior algebra over $\mathbb{Q}$ on generators $a, x$ and $y$ of degrees $\operatorname{deg}(a)=1, \operatorname{deg}(x)=\operatorname{deg}(y)=3$, and $S\left(w_{\ell}\right)$ denotes the polynomial algebra over $\mathbb{Q}$ on the generator $w_{\ell}$ of degree $4 \ell$.

\section{Hamiltonian Torus Actions}

In this section we define Hamiltonian torus actions on symplectic manifolds and present the Atiyah-Guillemin-Sternberg's Convexity Theorem for their moment maps. We also briefly discuss symplectic toric orbifolds and their classification due to Delzant and Lerman-Tolman.

Definition and basic examples. Let $(M, \omega)$ be a symplectic manifold equipped with a symplectic action of

$$
\mathbb{T}^{m} \equiv \mathbb{R}^{m} / 2 \pi \mathbb{Z}^{m} \equiv \mathbb{R} / 2 \pi \mathbb{Z} \times \cdots \times \mathbb{R} / 2 \pi \mathbb{Z} \equiv S^{1} \times \cdots \times S^{1},
$$

i.e. with a homomorphism $\mathbb{T}^{m} \rightarrow \operatorname{Diff}(M, \omega)$. Let $X_{1}, \ldots, X_{m} \in \mathcal{X}(M)$ be the vector fields generating the action of each individual $S^{1}$-factor. Then, since the action is symplectic, we have that

$$
\begin{gathered}
\left.\left.\left.\mathcal{L}_{X_{k}} \omega=0 \Leftrightarrow X_{k}\right\lrcorner d \omega+d\left(X_{k}\right\lrcorner \omega\right)=0 \Leftrightarrow d\left(X_{k}\right\lrcorner \omega\right)=0, \text { i.e. } \\
X_{k} \in \mathcal{X}(M, \omega), \forall k \in\{1, \ldots, m\} .
\end{gathered}
$$

Definition 4.1. A symplectic $\mathbb{T}^{m}$-action on a symplectic manifold $(M, \omega)$ is said to be Hamiltonian if for every $k \in\{1, \ldots, m\}$ there exists a function $h_{k}: M \rightarrow \mathbb{R}$ such that $\left.X_{k}\right\lrcorner \omega=d h_{k}$, i.e. $X_{k} \equiv X_{h_{k}} \in \mathcal{X}_{H}(M, \omega)$ is the Hamiltonian vector field of $h_{k}$. In this case, the map $\mu: M \rightarrow \mathbb{R}^{m}$ defined by

$$
\mu(p)=\left(h_{1}(p), \ldots, h_{m}(p)\right), \forall p \in M,
$$


is called a moment map for the action.

Remark 4.2. Suppose $\mu: M \rightarrow \mathbb{R}^{m}$ is a moment map for a Hamiltonian $\mathbb{T}^{m}$-action on $(M, \omega)$. Then $\mu+c$, for any given constant $c \in \mathbb{R}^{m}$, is also a moment map for that same action.

Remark 4.3. The orbits of a Hamiltonian $\mathbb{T}^{m}$-action on a symplectic manifold $(M, \omega)$ are always isotropic, i.e.

$$
\left.\omega\right|_{\text {orbit }} \equiv 0 \text {. }
$$

Example 4.4. The standard $\mathbb{T}^{n}$-action on $\left(\mathbb{R}^{2 n}, \omega_{\text {st }}\right)$ is Hamiltonian with moment map $\mu: \mathbb{R}^{2 n} \rightarrow \mathbb{R}^{n}$ given by

$$
\mu\left(x_{1}, \ldots, x_{n}, y_{1}, \ldots, y_{n}\right)=-\frac{1}{2}\left(x_{1}^{2}+y_{1}^{2}, \ldots, x_{n}^{2}+y_{n}^{2}\right) .
$$

Example 4.5. Consider the 2-sphere

$$
S^{2}=\left\{(x, y, z) \in \mathbb{R}^{3}: x^{2}+y^{2}+z^{2}=1\right\} \subset \mathbb{R}^{3}
$$

with symplectic or area form $4 \pi \sigma$ induced by the standard euclidean inner product in $\mathbb{R}^{3}$. The height function $h: S^{2} \rightarrow \mathbb{R}$, given by $h(x, y, z)=$ $z$, generates through its Hamiltonian vector field $X_{h}$ the rotations of $S^{2}$ around its vertical axis. Hence, this is an example of a Hamiltonian $S^{1}$ action on $\left(S^{2}, 4 \pi \sigma\right)$ with moment map $\mu \equiv h$.

In the last 25 years an incredible amount of research has been devoted to the study of moment maps and their beautiful geometric properties. We will now present two of these.

Atiyah-Guillemin-Sternberg's Convexity Theorem. Atiyah [9] and Guillemin-Sternberg [25] proved in 1982 the following Convexity Theorem.

Theorem 4.6. Let $(M, \omega)$ be a compact, connected, symplectic manifold, equipped with a Hamiltonian $\mathbb{T}^{m}$-action with moment map $\mu: M \rightarrow \mathbb{R}^{m}$. Then

(i) the level sets $\mu^{-1}(\lambda)$ of the moment map are connected (for any $\left.\lambda \in \mathbb{R}^{m}\right)$;

(ii) the image $\mu(M) \subset \mathbb{R}^{m}$ of the moment map is the convex hull of the images of the fixed points of the action.

The image $\mu(M) \subset \mathbb{R}^{m}$ of the moment map is called the moment polytope.

Example 4.7. In Example 4.5, the fixed points of the $S^{1}$-action are the poles $S=(0,0,-1)$ and $N=(0,0,1)$ of the 2-sphere $S^{2} \subset \mathbb{R}^{3}$. The images of these fixed points under the moment map are $\mu(S)=-1$ and $\mu(N)=1$, while the moment polytope is $\mu\left(S^{2}\right)=[-1,1] \subset \mathbb{R}$. 
Symplectic toric manifolds. The following proposition motivates the definition of a symplectic toric manifold.

Proposition 4.8. If a symplectic manifold $(M, \omega)$ has an effective Hamiltonian $\mathbb{T}^{m}$-action, then $m \leq(\operatorname{dim} M) / 2$.

Proof.

Effective action $\Rightarrow$ there exist $m$-dimensional orbits.

Hamiltonian $\mathbb{T}^{m}$-action $\Rightarrow$ orbits are isotropic (see Remark 4.3).

Linear Algebra $\Rightarrow \operatorname{dim}($ isotropic orbit $) \leq \frac{1}{2} \operatorname{dim} M$.

Definition 4.9. A symplectic toric manifold is a connected symplectic manifold $(M, \omega)$ of dimension $2 n$ equipped with an effective Hamiltonian $\mathbb{T}^{n}$-action.

Example 4.10. $\left(S^{2}, 4 \pi \sigma\right)$, with the $S^{1}$-action decribed in Example 4.5, is the simplest compact symplectic toric manifold.

Example 4.11. $\left(\mathbb{R}^{2 n}, \omega_{\mathrm{st}}\right)$, with its standard Hamiltonian $\mathbb{T}^{n}$-action (Example 4.4), is a non-compact symplectic toric manifold.

Example 4.12. The $\mathbb{T}^{n}$-action on $\left(\mathbb{C P}^{n}, \omega_{F S}\right)$ given in homogeneous coordinates by

$$
\left(\theta_{1}, \ldots, \theta_{n}\right) \cdot\left[z_{0} ; z_{1} ; \ldots ; z_{n}\right]=\left[z_{0} ; e^{i \theta_{1}} z_{1} ; \ldots ; e^{i \theta_{n}} z_{n}\right]
$$

is Hamiltonian, with moment map $\mu: \mathbb{C P}^{n} \rightarrow \mathbb{R}^{n}$ given by

$$
\mu\left(\left[z_{0} ; z_{1} ; \ldots ; z_{n}\right]\right)=-\frac{1}{\|z\|^{2}}\left(\left\|z_{1}\right\|^{2}, \ldots,\left\|z_{n}\right\|^{2}\right)
$$

where $\|z\|^{2}=\left\|z_{0}\right\|^{2}+\left\|z_{1}\right\|^{2}+\cdots+\left\|z_{n}\right\|^{2}$. Hence, $\left(\mathbb{C P}^{n}, \omega_{F S}\right)$ equipped with this $\mathbb{T}^{n}$-action is a compact symplectic toric manifold. Note that its moment polytope is a simplex in $\mathbb{R}^{n}$.

It follows from Theorem 4.6 that any compact symplectic toric manifold has an associated convex polytope, the moment polytope of the torus action. In 1988 Delzant [16] characterized the convex polytopes that arise as moment map images of compact symplectic toric manifolds, and showed that any such convex polytope determines a unique compact symplectic toric manifold. More precisely, if two compact symplectic toric manifolds have the same moment polytope, then there exists an equivariant symplectomorphism between them. Delzant's result can be summarized in the following theorem.

Theorem 4.13. [Delzant] The moment polytope is a complete invariant of a compact symplectic toric manifold. 
Symplectic toric orbifolds. In [27] Lerman and Tolman generalize Theorems 4.6 and 4.13 to orbifolds. While the convexity theorem generalizes word for word, one needs more information than just the convex polytope $P$ to generalize Delzant's classification theorem.

Definition 4.14. A convex polytope $P$ in $\left(\mathbb{R}^{n}\right)^{*}$ is called simple and rational if:

(1) there are $n$ edges meeting at each vertex $p$;

(2) the edges meeting at the vertex $p$ are rational, i.e. each edge is of the form $p+t v_{i}, 0 \leq t \leq \infty$, where $v_{i} \in\left(\mathbb{Z}^{n}\right)^{*}$;

(3) the $v_{1}, \ldots, v_{n}$ in (2) can be chosen to be a $\mathbb{Q}$-basis of the lattice $\left(\mathbb{Z}^{n}\right)^{*}$.

$A$ facet is a face of $P$ of codimension one. Following Lerman-Tolman, we will say that a labeled polytope is a rational simple convex polytope $P \subset\left(\mathbb{R}^{n}\right)^{*}$, plus a positive integer (label) attached to each of its facets.

Two labeled polytopes are isomorphic if one can be mapped to the other by a translation, and the corresponding facets have the same integer labels.

Remark 4.15. In Delzant's classification theorem for compact symplectic toric manifolds, there are no labels (or equivalently, all labels are equal to 1 ) and the polytopes that arise are slightly more restrictive: the "Q্Q" in (3) is replaced by " $\mathbb{Z}$ ".

Theorem 4.16 (Lerman-Tolman). Let $(M, \omega)$ be a compact symplectic toric orbifold, with moment map $\mu: M \rightarrow\left(\mathbb{R}^{n}\right)^{*}$. Then $P \equiv \mu(M)$ is a rational simple convex polytope. For every facet $F$ of $P$, there exists a positive integer $m_{F}$, the label of $F$, such that the orbifold structure group of every $p \in \mu^{-1}(\breve{F})$ is $\mathbb{Z} / m_{F} \mathbb{Z}$ (here $\breve{F}$ is the relative interior of $F$ ).

Two compact symplectic toric orbifolds are equivariant symplectomorphic (with respect to a fixed torus acting on both) if and only if their associated labeled polytopes are isomorphic. Moreover, every labeled polytope arises from some compact symplectic toric orbifold.

\section{Kähler geometry of toric orbifolds in symplectic coordi- nates}

The space of Kähler metrics on a Kähler manifold (or orbifold) can be described in two equivalent ways, reflecting the fact that a Kähler manifold is both a complex and a symplectic manifold.

From the complex point of view, one starts with a fixed complex manifold $\left(M, J_{0}\right)$ and Kähler class $\Omega \in H_{J_{0}}^{1,1} \cap H^{2}(M, \mathbb{R})$, and considers the space $\mathcal{S}\left(J_{0}, \Omega\right)$ of all symplectic forms $\omega$ on $M$ that are compatible with $J_{0}$ and 
represent the class $\Omega$. Any such form $\omega \in \mathcal{S}\left(J_{0}, \Omega\right)$ gives rise to a Kähler metric $\langle\cdot, \cdot\rangle \equiv \omega\left(\cdot, J_{0} \cdot\right)$.

The symplectic point of view arises naturally from the observation that any two forms $\omega_{0}, \omega_{1} \in \mathcal{S}\left(J_{0}, \Omega\right)$ define equivalent symplectic structures on $M$. In fact, the family $\omega_{t}=\omega_{0}+t\left(\omega_{1}-\omega_{0}\right)$, for $t \in[0,1]$, is an isotopy of symplectic forms in the same cohomology class $\Omega$, and so Moser's theorem [30] gives a family of diffeomorphisms $\varphi_{t}: M \rightarrow M, t \in[0,1]$, such that $\varphi_{t}^{*}\left(\omega_{t}\right)=\omega_{0}$. In particular, the Kähler manifold $\left(M, J_{0}, \omega_{1}\right)$ is Kähler isomorphic to $\left(M, J_{1}, \omega_{0}\right)$, where $J_{1}=\left(\varphi_{1}\right)_{*}^{-1} \circ J_{0} \circ\left(\varphi_{1}\right)_{*}$.

This means that one can also describe the space of Kähler metrics starting with a fixed symplectic manifold $\left(M, \omega_{0}\right)$ and considering the space $\mathcal{J}\left(\omega_{0},\left[J_{0}\right]\right)$ of all complex structures $J$ on $M$ that are compatible with $\omega_{0}$ and belong to some diffeomorphism class $\left[J_{0}\right]$, determined by a particular compatible complex structure $J_{0}$. Any such $J \in \mathcal{J}\left(\omega_{0},\left[J_{0}\right]\right)$ gives rise to a Kähler metric $\langle\cdot, \cdot\rangle \equiv \omega_{0}(\cdot, J \cdot)$.

Apriori, the symplectic point of view does not seem to be very effective for solving specific problems in Kähler geometry, the reason being that the space $\mathcal{J}\left(\omega_{0},\left[J_{0}\right]\right)$ is non-linear and difficult to parametrize. The complex point of view fairs much better in this regard, since the space $\mathcal{S}\left(J_{0}, \Omega\right)$ can be identified with an open convex subset of the linear space of smooth functions on $M$. Indeed, the $\partial \bar{\partial}$-lemma asserts that given $\omega_{0} \in \mathcal{S}\left(J_{0}, \Omega\right)$ any other $\omega \in \mathcal{S}\left(J_{0}, \Omega\right)$ can be written as

$$
\omega=\omega_{0}+2 i \partial \bar{\partial} f \text {, for some } f \in C^{\infty}(M) .
$$

Moreover, the set of functions $f \in C^{\infty}(M)$ for which the form $\omega$ defined by (3) is in $\mathcal{S}\left(J_{0}, \Omega\right)$ is open and convex.

There are however particular situations in which the space $\mathcal{J}\left(\omega_{0},\left[J_{0}\right]\right)$ admits a parametrization similar to the one just described for $\mathcal{S}\left(J_{0}, \Omega\right)$, and the symplectic point of view can then be used very effectively. Following Guillemin [24] and [3, 4, 5] we will now describe how that is indeed the case for Kähler toric orbifolds.

Symplectic Potential. Let $(M, \omega)$ be a symplectic toric orbifold of dimension $2 n$, equipped with an effective Hamiltonian action $\tau: \mathbb{T}^{n} \rightarrow$ $\operatorname{Diff}(M, \omega)$ of the standard (real) $n$-torus $\mathbb{T}^{n}=\mathbb{R}^{n} / 2 \pi \mathbb{Z}^{n}$, i.e. $(M, \omega, \tau)$ is a symplectic toric orbifold. Denote by $\mu: M \rightarrow\left(\mathbb{R}^{n}\right)^{*}$ the moment map of such an action. Theorem 4.16 then says that $P \equiv \mu(M) \subset\left(\mathbb{R}^{n}\right)^{*}$ is a convex rational simple polytope that, together with a positive integer label attached to each of its facets, completely determines the symplectic toric orbifold. 
The proof gives an explicit construction of a canonical model for each symplectic toric orbifold, i.e. it associates to each labeled polytope $P$ an explicit symplectic toric orbifold $\left(M_{P}, \omega_{P}, \tau_{P}\right)$ with moment map $\mu_{P}: M_{P} \rightarrow$ $P$. Moreover, it follows from the construction that $M_{P}$ has a canonical $\mathbb{T}^{n}$-invariant complex structure $J_{P}$ compatible with $\omega_{P}$. In other words, associated to each labeled polytope $P \subset\left(\mathbb{R}^{n}\right)^{*}$ one has a canonical Kähler toric orbifold $\left(M_{P}, \omega_{P}, J_{P}, \tau_{P}\right)$ with moment map $\mu_{P}: M_{P} \rightarrow P$.

The symplectic description of compatible toric complex structures and Kähler metrics is based on the following set-up (see [4] for details). Let $\breve{P}$ denote the interior of $P$, and consider $\breve{M}_{P} \subset M_{P}$ defined by $\breve{M}_{P}=$ $\mu_{P}^{-1}(\breve{P})$. One can easily check that $\breve{M}_{P}$ is a smooth open dense subset of $M_{P}$, consisting of all the points where the $\mathbb{T}^{n}$-action is free. It can be described as

$$
\breve{M}_{P} \cong \breve{P} \times \mathbb{T}^{n}=\left\{(x, \theta): x \in \breve{P} \subset\left(\mathbb{R}^{n}\right)^{*}, \theta \in \mathbb{R}^{n} / 2 \pi \mathbb{Z}^{n}\right\},
$$

where $(x, \theta)$ are symplectic (or action-angle) coordinates for $\omega_{P}$, i.e.

$$
\omega_{P}=d x \wedge d \theta=\sum_{j=1}^{n} d x_{j} \wedge d \theta_{j} .
$$

If $J$ is any $\omega_{P}$-compatible toric complex structure on $M_{P}$, the symplectic $(x, \theta)$-coordinates on $\breve{M}_{P}$ can be chosen so that the matrix that represents $J$ in these coordinates has the form

$$
\left[\begin{array}{ccc}
0 & \vdots & -G^{-1} \\
\ldots \ldots & \ldots
\end{array}\right]
$$

where $G=G(x)=\left[g_{j k}(x)\right]_{j, k=1}^{n, n}$ is a symmetric and positive-definite real matrix. The integrability condition for the complex structure $J$ is equivalent to $G$ being the Hessian of a smooth function $g \in C^{\infty}(\breve{P})$, i.e.

$$
G=\operatorname{Hess}_{x}(g), g_{j k}(x)=\frac{\partial^{2} g}{\partial x_{j} \partial x_{k}}(x), 1 \leq j, k \leq n .
$$

Holomorphic coordinates for $J$ are given in this case by

$$
z(x, \theta)=u(x, \theta)+i v(x, \theta)=\frac{\partial g}{\partial x}(x)+i \theta .
$$

We will call $g$ the symplectic potential of the compatible toric complex structure $J$. Note that the Kähler metric $\langle\cdot, \cdot\rangle=\omega_{P}(\cdot, J \cdot)$ is given in these 
$(x, \theta)$-coordinates by the matrix

$$
\left[\begin{array}{ccc}
G & \vdots & 0 \\
\cdots & \cdots & \cdots \\
0 & \vdots & G^{-1}
\end{array}\right]
$$

In particular, the induced metric on any slice of the form $\breve{P} \times\{$ point $\} \subset \breve{M}_{P}$ is given by the matrix $G$.

Every convex rational simple polytope $P \subset\left(\mathbb{R}^{n}\right)^{*}$ can be described by a set of inequalities of the form

$$
\left\langle x, \mu_{r}\right\rangle \geq \rho_{r}, r=1, \ldots, d,
$$

where $d$ is the number of facets of $P$, each $\mu_{r}$ is a primitive element of the lattice $\mathbb{Z}^{n} \subset \mathbb{R}^{n}$ (the inward-pointing normal to the $r$-th facet of $\mathrm{P}$ ), and each $\rho_{r}$ is a real number. The labels $m_{r} \in \mathbb{N}$ attached to the facets can be incorporated in the description of $P$ by considering the affine functions $\ell_{r}:\left(\mathbb{R}^{n}\right)^{*} \rightarrow \mathbb{R}$ defined by

$$
\ell_{r}(x)=\left\langle x, m_{r} \mu_{r}\right\rangle-\lambda_{r} \text { where } \lambda_{r}=m_{r} \rho_{r} \text { and } r=1, \ldots, d .
$$

Then $x$ belongs to the $r$-th facet of $P$ iff $\ell_{r}(x)=0$, and $x \in \breve{P}$ iff $\ell_{r}(x)>0$ for all $r=1, \ldots, d$.

The following two theorems are proved in [5]. The first is a straightforward generalization to toric orbifolds of a result of Guillemin [24].

Theorem 5.1. Let $\left(M_{P}, \omega_{P}, \tau_{P}\right)$ be the symplectic toric orbifold associated to a labeled polytope $P \subset\left(\mathbb{R}^{n}\right)^{*}$. Then, in suitable symplectic $(x, \theta)$ coordinates on $\breve{M}_{P} \cong \breve{P} \times \mathbb{T}^{n}$, the canonical compatible toric complex structure $J_{P}$ is of the form (4)-(5) for a potential $g_{P} \in C^{\infty}(\breve{P})$ given by

$$
g_{P}(x)=\frac{1}{2} \sum_{r=1}^{d} \ell_{r}(x) \log \ell_{r}(x) .
$$

The second theorem provides the symplectic version of (3) in this toric orbifold context, generalizing an analogous result for toric manifolds proved in [4].

Theorem 5.2. Let $J$ be any compatible toric complex structure on the symplectic toric orbifold $\left(M_{P}, \omega_{P}, \tau_{P}\right)$. Then, in suitable symplectic $(x, \theta)$ coordinates on $\breve{M}_{P} \cong \breve{P} \times \mathbb{T}^{n}$, $J$ is given by (4)-(5) for a potential $g \in$ $C^{\infty}(\breve{P})$ of the form

$$
g(x)=g_{P}(x)+h(x)
$$


where $g_{P}$ is given by Theorem 5.1, $h$ is smooth on the whole $P$, and the matrix $G=\operatorname{Hess}(g)$ is positive definite on $\breve{P}$ and has determinant of the form

$$
\operatorname{Det}(G)=\left(\delta \prod_{r=1}^{d} \ell_{r}\right)^{-1},
$$

with $\delta$ being a smooth and strictly positive function on the whole $P$.

Conversely, any such potential $g$ determines by (4)-(5) a complex structure on $\breve{M}_{P} \cong \breve{P} \times \mathbb{T}^{n}$, that compactifies to a well-defined compatible toric complex structure $J$ on the symplectic toric orbifold $\left(M_{P}, \omega_{P}, \tau_{P}\right)$.

Note that there is no imposed condition of $J$ being in the same diffeomorphism class as $J_{P}$. The reason is that, by Theorem 9.4 in [27], any compatible toric $J$ on $\left(M_{P}, \omega_{P}, \tau_{P}\right)$ is equivariantly biholomorphic to $J_{P}$.

Scalar Curvature. In [12] and [13], Calabi introduced the notion of extremal Kähler metrics. These are defined, for a fixed closed complex manifold $\left(M, J_{0}\right)$, as critical points of the square of the $L^{2}$-norm of the scalar curvature, considered as a functional on the space of all symplectic Kähler forms $\omega$ in a fixed Kähler class $\Omega \in H^{2}(M, \mathbb{R})$. The extremal EulerLagrange equation is equivalent to the gradient of the scalar curvature being an holomorphic vector field (see [12]), and so these metrics generalize constant scalar curvature Kähler metrics. Moreover, Calabi showed in [13] that extremal Kähler metrics are always invariant under a maximal compact subgroup of the group of holomorphic transformations of $\left(M, J_{0}\right)$. Hence, on a complex toric manifold or orbifold, extremal Kähler metrics are automatically toric Kähler metrics, and one should be able to write them down using the previous symplectic framework.

Before doing that for a specific class of explicit examples, we now recall from [3] some relevant differential-geometric formulas in symplectic $(x, \theta)$ coordinates. A Kähler metric of the form (6) has scalar curvature $S$ given by $^{1}$

$$
S=-\sum_{j, k} \frac{\partial}{\partial x_{j}}\left(g^{j k} \frac{\partial \log \operatorname{Det}(G)}{\partial x_{k}}\right),
$$

which after some algebraic manipulations becomes the more compact

$$
S=-\sum_{j, k} \frac{\partial^{2} g^{j k}}{\partial x_{j} \partial x_{k}},
$$

\footnotetext{
${ }^{1}$ The normalization for the value of the scalar curvature we are using here is the same as in [10]. It differs from the one used in $[3,4]$ by a factor of $1 / 2$.
} 
where the $g^{j k}, 1 \leq j, k \leq n$, are the entries of the inverse of the matrix

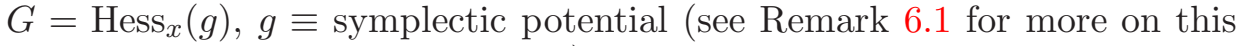
expression for the scalar curvature). The Euler-Lagrange equation defining an extremal Kähler metric can be shown to be equivalent to

$$
\frac{\partial S}{\partial x_{j}} \equiv \text { constant }, j=1, \ldots, n,
$$

i.e. the metric is extremal if and only if its scalar curvature $S$ is an affine function of $x$.

Remark 5.3. The general nonlinear, fourth order, partial differential equation for a convex function $u$ on an open set $\Omega$ in $\mathbb{R}^{n}$, given by

$$
-\sum_{j, k} \frac{\partial^{2} u^{j k}}{\partial x_{j} \partial x_{k}}=A
$$

where $A$ is some given function, is studied by Donaldson in [20, 21, 22].

Extremal Metrics on Weighted Projective Spaces. In [11] R. Bryant studies and classifies Bochner-Kähler metrics, i.e. Kähler metrics with vanishing Bochner curvature. He shows in particular that these metrics always have a very high degree of symmetry, the least symmetric ones being of toric type. It turns out that the models for these least symmetric BochnerKähler metrics, given by Theorem 9 in [11], have a very simple explicit description in the above symplectic framework. For us, their most relevant geometric property is that of being extremal and, following [5], we will describe them only as such.

Let $P_{\mathbf{m}}^{n}$ denote the labeled simplex in $\left(\mathbb{R}^{n}\right)^{*}$ defined by the affine functions

$$
\ell_{r}(x)=m_{r}\left(1+x_{r}\right), r=1, \ldots, n, \ell_{n+1}(x)=m_{n+1}(1-\psi), \psi=\sum_{j=1}^{n} x_{j}
$$

where $\mathbf{m}=\left(m_{1}, \ldots, m_{n+1}\right) \in \mathbb{N}^{n+1}$ is a vector of positive integer labels. The associated symplectic toric orbifold will be called a labeled projective space and denoted by $\left(\mathbb{S P}_{\mathbf{m}}^{n}, \omega_{\mathbf{m}}, \tau_{\mathbf{m}}\right)$ ( the "S" is supposed to emphasize its $\mathbb{S} y m p l e c t i c$ nature).

Theorem 5.4. For any vector of labels $\mathbf{m} \in \mathbb{N}^{n+1}$, the potential $g \in$ $C^{\infty}\left(\breve{P}_{\mathbf{m}}^{n}\right)$ defined by

$$
g(x)=\frac{1}{2}\left(\sum_{r=1}^{n+1} \ell_{r}(x) \log \ell_{r}(x)-\ell_{\Sigma}(x) \log \ell_{\Sigma}(x)\right),
$$


where the $\ell_{r}$ 's are given by (10) and

$$
\ell_{\Sigma}(x)=\sum_{r=1}^{n+1} \ell_{r}(x),
$$

gives rise to an extremal compatible toric complex structure on $\left(\mathbb{S P}_{\mathbf{m}}^{n}, \omega_{\mathbf{m}}, \tau_{\mathbf{m}}\right)$. In other words, the metric defined by (6) is an extremal Kähler metric.

There is a close relation between labeled projective spaces $\mathbb{S P}_{\mathbf{m}}^{n}$ and the more common weighted projective spaces $\mathbb{C P}_{\text {a }}^{n}$ (see [5]). These are defined for a given vector of positive integer weights $\mathbf{a}=\left(a_{1}, \ldots, a_{n+1}\right) \in \mathbb{N}^{n+1}$ as

$$
\mathbb{C P}_{\mathbf{a}}^{n} \equiv\left(\mathbb{C}^{n+1} \backslash\{0\}\right) / \mathbb{C}^{*},
$$

where the action of $\mathbb{C}^{*}=\mathbb{C} \backslash\{0\}$ on $\mathbb{C}^{n+1}$ is given by

$$
\left(z_{1}, \ldots, z_{n+1}\right) \stackrel{t}{\mapsto}\left(t^{a_{1}} z_{1}, \ldots, t^{a_{n+1}} z_{n+1}\right), t \in \mathbb{C}^{*} .
$$

The relation between $\mathbb{S P}_{\mathbf{m}}^{n}$ and $\mathbb{C P}_{\mathbf{m}}^{n}$ implies the following corollary to Theorem 5.4 (see also Theorem 11 in [11]).

Corollary 5.5. Every weighted projective space $\mathbb{C P}_{\mathbf{a}}^{n}$ has an extremal Kähler metric.

\section{Moment Map Geometry}

In this section, following [17], we recall a general moment map framework and how it applies to the action of a symplectomorphism group on the corresponding space of compatible almost-complex structures.

General Framework. Let $G$ be a Lie group, $\mathcal{G}$ its Lie algebra, $\langle\cdot, \cdot\rangle$ an inner product on $\mathcal{G}$ invariant under the adjoint action, $\mathcal{G}^{*}$ the dual Lie algebra naturally identified with $\mathcal{G}$ via $\langle\cdot, \cdot\rangle$, and $G^{\mathbb{C}}$ a complexification of $G$.

Let $(X, J, \Omega)$ be a Kähler manifold equipped with an action of $G$ by Kähler isometries, i.e. a homomorphism

$$
\rho: G \rightarrow \operatorname{Iso}(X, J, \Omega)=\operatorname{Hol}(X, J) \cap \operatorname{Symp}(X, \Omega) .
$$

Suppose this action satisfies the following two conditions:

(i) the holomorphic action of $G$ on $(X, J)$ extends to a holomorphic action of $G^{\mathbb{C}}$ on $(X, J)$;

(ii) the symplectic action of $G$ on $(X, \Omega)$ admits a suitably normalized equivariant moment map $\mu: X \rightarrow \mathcal{G}^{*}$. 
Then we have the following two general principles.

General Principle I. The complex and symplectic quotients of $X$ by $G$ are naturally identified. More precisely,

$$
\mu^{-1}(0) / G=X^{s} / G^{\mathbb{C}}
$$

where $X^{s} \subset X$ is an open, $G^{\mathbb{C}}$-invariant, subset of "stable points". We will not define here this notion of stability, the important point being that it should only depend on the holomorphic geometry of the situation. The content of this principle is that on each stable $G^{\mathbb{C}}$-orbit there is a point $p \in \mu^{-1}(0)$, unique up to the action of $G$.

General Principle II. The map $\|\mu\|^{2} \equiv\langle\mu, \mu\rangle: X \rightarrow \mathbb{R}$ behaves like a $G$-invariant Morse-Bott function, whose critical manifolds compute the equivariant cohomology $H_{G}^{*}(X) \equiv H^{*}\left(X \times_{G} E G\right)$ (over $\mathbb{Q}$ and, in good special cases, also over $\mathbb{Z}$ ).

Combining these two general principles, one gets the following geometric picture for the action of $G$ on $X$ :

- The gradient flow of $-\|\mu\|^{2}$ induces an invariant stratification

$$
X=V_{0} \sqcup V_{1} \sqcup V_{2} \sqcup \cdots,
$$

where each $V_{k}$ is the stable manifold of some critical set $C_{k}$ of $\|\mu\|^{2}$.

- Let $\mathcal{O}_{k}$ denote the coadjoint orbit $G \cdot \xi_{k} \subset \mathcal{G}^{*}$, where $\xi_{k}=\mu\left(p_{k}\right)$ for some $p_{k} \in C_{k}$. Then

$$
V_{k} / G^{\mathbb{C}} \simeq \mu^{-1}\left(\mathcal{O}_{k}\right) / G
$$

If $C_{0}=\mu^{-1}(0)$ then $\mathcal{O}_{0}=\{0\}$ and $V_{0}=X^{s}$.

(1) - The equivariant cohomology $H_{G}^{*}(X)$ can be computed from $H_{G}^{*}\left(V_{k}\right), k=0,1,2, \ldots$ (over $\mathbb{Q}$ and, in good special cases, also over $\mathbb{Z})$.

\section{Symplectomorphism Groups and Compatible Complex}

Structures. Consider a compact symplectic manifold $(M, \omega)$, of dimension $2 n$, and assume that $H^{1}(M, \mathbb{R})=0$. Let $G \equiv \operatorname{Symp}(M, \omega)$ be the symplectomorphism group of $(M, \omega)$. This is an infinite dimensional Lie group whose Lie algebra $\mathcal{G}$ can be identified with the space of functions on $M$ with integral zero:

$$
\mathcal{G}=C_{0}^{\infty}(M) \equiv\left\{f: M \rightarrow \mathbb{R}: \int_{M} f \frac{\omega^{n}}{n !}=0\right\}
$$


$\mathcal{G}$ has a natural invariant inner product $\langle\cdot, \cdot\rangle$, given by

$$
\langle f, g\rangle \equiv \int_{M} f \cdot g \frac{\omega^{n}}{n !},
$$

which will be used to identify $\mathcal{G}^{*}$ with $\mathcal{G}$.

Consider now the space $\mathcal{J}(M, \omega)$ of almost complex structures $J$ on $M$ which are compatible with $\omega$, i.e. for which the bilinear form

$$
g_{J}(\cdot, \cdot)=\omega(\cdot, J \cdot)
$$

is a Riemannian metric on $M$. This is the space of sections of a bundle over $M$ with fiber the contractible symmetric Kähler manifold $\operatorname{Sp}(2 n, \mathbb{R}) / U(n) \equiv$ Siegel upper half space [32]. This fiberwise symmetric Kähler structure, together with the volume form induced by $\omega$ on $M$, turns $\mathcal{J}(M, \omega)$ into an infinite dimensional (contractible) Kähler manifold.

The symplectomorphism group $G$ acts naturally on $\mathcal{J}(M, \omega)$ by Kähler isometries:

$$
\phi \cdot J \equiv \phi_{*}(J)=d \phi \circ J \circ d \phi^{-1}, \forall \phi \in G, J \in \mathcal{J}(M, \omega) .
$$

To fit the previous general framework, this action should satisfy conditions (i) and (ii).

The first (holomorphic) condition poses an immediate problem since there is no complexification $G^{\mathbb{C}}$ of the symplectomorphism group $G$. However, we can certainly complexify the Lie algebra $\mathcal{G}$ to

$$
\mathcal{G}^{\mathbb{C}} \equiv\left\{f: M \rightarrow \mathbb{C}: \int_{M} f \frac{\omega^{n}}{n !}=0\right\}
$$

and the infinitesimal action of $\mathcal{G}$ on $\mathcal{J}(M, \omega)$ extends to an action of $\mathcal{G}^{\mathbb{C}}$, since the complex structure on $\mathcal{J}(M, \omega)$ is integrable. This gives rise to an integrable complex distribution on $\mathcal{J}(M, \omega)$ whose leaves play the role of "connected components of orbits of the group $G^{\mathbb{C}}$ ".

In the holomorphic side of General Principles I and II that we want to apply, $G^{\mathbb{C}}$ is not that important when compared with the role played by its orbits. The geometric meaning of these " $G$ - ${ }^{\mathbb{C}}$-orbits" becomes quite clear if one restricts the actions under consideration to the invariant Kähler submanifold $X$ of compatible integrable complex structures

$$
X \equiv \mathcal{J}^{\text {int }}(M, \omega) \subset \mathcal{J}(M, \omega),
$$

determined by the vanishing of the Nijenhuis tensor. Here, it follows from Donaldon's analysis in [17] that

$J, J^{\prime} \in X$ belong to the same " $G^{\mathbb{C}}$-orbit" iff there exists $\varphi \in \operatorname{Diff}(M)$ such that

$$
\left[\varphi^{*}(\omega)\right]=[\omega] \in H^{2}(M, \mathbb{R}) \quad \text { and } \quad \varphi_{*}(J)=J^{\prime} .
$$


This explicit description of a " $G$ C-orbit" is good enough to consider that the holomorphic action of $G=\operatorname{Symp}(M, \omega)$ on $X=\mathcal{J}^{\text {int }}(M, \omega)$ satisfies condition (i).

Regarding condition (ii), Donaldson [17] shows that there always exists an equivariant and suitably normalized moment map

$$
\mu: \mathcal{J}(M, \omega) \rightarrow \mathcal{G}^{*} \cong C_{0}^{\infty}(M)
$$

for the symplectic action of $G$ on $\mathcal{J}(M, \omega)$, given by

$$
\mu(J)=\left(\text { Hermitian scalar curvature } S(J) \text { of the metric } g_{J}\right)-d,
$$

where $d$ is the constant defined by

$$
d \cdot \int_{M} \frac{\omega^{n}}{n !} \equiv 2 \pi c_{1}(M) \wedge[\omega]^{n-1}(M)=\int_{M} S(J) \frac{\omega^{n}}{n !} .
$$

Note that on $X \subset \mathcal{J}(M, \omega)$, i.e. for integrable $J$, the Hermitian scalar curvature $S(J)$ coincides with the usual scalar curvature of the Riemannian metric $g_{J}$.

Remark 6.1. The symplectic approach to Kähler geometry of toric manifolds presented in section 5 fits very well with this framework. For example, Donaldson shows in [20] that, viewed as the moment map for the action described above, the natural expression for the scalar curvature is given by (8).

We have concluded that the Kähler action of $G$ on $X$ satisfies conditions (i) and (ii) of the general framework, and so General Principles I and II should apply. What do they say in this context?

General Principle I says that each stable compatible complex structure is diffeomorphic to one in $\mu^{-1}(0)$, unique up to the action of $G$. Since

$$
J \in \mu^{-1}(0) \Leftrightarrow \mu(J)=0 \Leftrightarrow S(J)=d=\text { constant }
$$

this says that on each diffeomorphism class of stable compatible complex structures there should exist a unique $\operatorname{Symp}(M, \omega)$-orbit whose corresponding Kähler metric has constant scalar curvature. (See the work of Donaldson [18], [19] and [20], exploring this consequence of General Principle I.)

General Principle II says that the critical points of

$$
\|\mu\|^{2}: X=\mathcal{J}^{\operatorname{int}}(M, \omega) \rightarrow \mathbb{R},\|\mu\|^{2}(J)=\int_{M} S^{2}(J) \frac{\omega^{n}}{n !}+\text { constant },
$$

determine the equivariant cohomology $H_{G}^{*}(X)$.

These critical points are, in particular, extremal Kähler metrics in the sense of Calabi ([12] and [13]). When extremal Kähler metrics exist, they 
minimize $\|\mu\|^{2}$ on the corresponding " $G^{\mathbb{C}}$-orbit" (see [26]) and are conjecturally unique up to the action of $G$ (see [14]).

In the concrete examples we will discuss (rational ruled surfaces) these general principles do hold. Whenever this is the case, one gets the following geometric picture for the action of $G=\operatorname{Symp}(M, \omega)$ on $X=\mathcal{J}^{\text {int }}(M, \omega)$ :

- There is a stratification of $X$ of the form

$$
X=V_{0} \sqcup V_{1} \sqcup V_{2} \sqcup \cdots .
$$

In this stratification, each $V_{k}$ contains the set of compatible complex structures which are diffeomorphic to an extremal one with normalized scalar curvature in

$$
\mathcal{O}_{k} \equiv \text { coadjoint orbit } G \cdot\left(S\left(J_{k}\right)-d\right) \text { in } C_{0}^{\infty}(M) \cong \mathcal{G}^{*},
$$

where $J_{k}$ is some critical compatible complex structure in $V_{k}$.

- " $V_{k} / G^{\mathbb{C} "} \equiv\left\{\right.$ " $G^{\mathbb{C}}$-orbits" in $\left.V_{k}\right\} \simeq \mu^{-1}\left(\mathcal{O}_{k}\right) / G$ is some moduli space of complex structures, that one might try to understand using methods from complex geometry (deformation theory).

- Let $\mathcal{O}_{J_{k}}$ denote the " $G \mathbb{C}^{\mathbb{C}}$-orbit" through some extremal $J_{k} \in V_{k}$ and let $K_{k} \equiv \operatorname{Iso}\left(M, \omega, J_{k}\right) \subset G$. Then, if the group $\operatorname{Hol}_{[\omega]}\left(M, J_{k}\right)$ of holomorphic automorphisms which preserve the cohomology class of $\omega$ is the complexification of $K_{k}$ (this is always the case if the groups are connected by [13]) then the inclusion

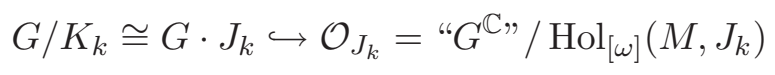

is a homotopy equivalence.

(1) -The equivariant cohomology $H_{G}^{*}(X)$ can be computed from $H_{G}^{*}\left(V_{k}\right), k=0,1,2, \ldots$ From the previous two points, each $H_{G}^{*}\left(V_{k}\right)$ should be determined from finite dimensional considerations involving moduli spaces of complex structures and subgroups of isometries in $G=\operatorname{Symp}(M, \omega)$. Recall that $\mathcal{J}(M, \omega)$ is always contractible. If $X=\mathcal{J}^{\operatorname{int}}(M, \omega) \subset \mathcal{J}(M, \omega)$ is also contractible, then

$$
H_{G}^{*}(X)=H^{*}(B G) \text {. }
$$

\section{Rational Ruled Surfaces}

In this section we discuss the particular case of rational ruled surfaces, formulating the precise results suggested by the framework of section 6 (see $[6,7]$ ). 
Symplectic Structures. As smooth 4-manifolds, rational ruled surfaces are $S^{2}$-bundles over $S^{2}$. Since $\pi_{2}\left(B \operatorname{Diff}^{+}\left(S^{2}\right)\right) \cong \pi_{2}(B S O(3)) \cong \mathbb{Z} / 2$, there are only two diffeomorphism types classified by the second StiefelWhitney class of the bundle (the mod 2 reduction of the Euler class): a trivial $S^{2}$-bundle over $S^{2}$ and a nontrivial $S^{2}$-bundle over $S^{2}$. Since the story for each of these is analogous, we will concentrate again on the trivial bundle, i.e.

$$
M=S^{2} \times S^{2} .
$$

Symplectic structures on $S^{2} \times S^{2}$ are classified by the following theorem.

Theorem 7.1. (Lalonde-McDuff) If $\omega$ is a symplectic form on $S^{2} \times S^{2}$, then it is diffeomorphic to $\lambda \sigma \oplus \mu \sigma$ for some real $\lambda, \mu>0$, where $\sigma$ denotes the standard area form on $S^{2}$ with $\int_{S^{2}} \sigma=1$.

Since the symplectomorphism group and the space of compatible almost complex structures is not affected by positive scalings of the symplectic form and we can switch the two $S^{2}$-factors, it will suffice to consider symplectic structures of the form

$$
\omega_{\lambda}=\lambda \sigma \oplus \sigma \quad \text { with } \quad 1 \leq \lambda \in \mathbb{R} .
$$

From now on we will use the following notation:

$$
\begin{aligned}
M_{\lambda} & =\left(S^{2} \times S^{2}, \omega_{\lambda}\right), 1 \leq \lambda \in \mathbb{R} ; \\
G_{\lambda} & =\operatorname{Symp}\left(M_{\lambda}\right)=\text { symplectomorphisms of } M_{\lambda} ; \\
\mathcal{J}_{\lambda} & =\mathcal{J}\left(M_{\lambda}\right)=\text { compatible almost complex structures; } \\
X_{\lambda} & =\mathcal{J}^{\text {int }}\left(M_{\lambda}\right)=\text { compatible integrable complex structures. }
\end{aligned}
$$

We will also use the following obvious isomorphism:

$$
\begin{aligned}
H_{2}\left(S^{2} \times S^{2}, \mathbb{Z}\right) & \cong \mathbb{Z} \oplus \mathbb{Z} \\
m\left[S^{2} \times \mathrm{pt}\right]+n\left[\mathrm{pt} \times S^{2}\right] & \longmapsto(m, n) .
\end{aligned}
$$

Compatible Integrable Complex Structures. As a complex manifold, a rational ruled surface is a holomorphic $\mathbb{C P}^{1}$-bundle over $\mathbb{C P}^{1}$. These are the well known Hirzebruch surfaces

$$
H_{k}=P(\mathcal{O} \oplus \mathcal{O}(-k)) \quad \text { for some } k \in \mathbb{N}_{0},
$$

where we write $\mathcal{O}(-1)$ for the tautological line bundle over $\mathbb{C P}^{1}$ and $P(E)$ for the projectivization of a vector bundle $E$.

Any complex structure $J$ on $S^{2} \times S^{2}$ is isomorphic to $H_{2 k}$ for some $k \in \mathbb{N}_{0}$, while the "odd" Hirzebruch surfaces are diffeomorphic to the nontrivial $S^{2}$-bundle over $S^{2}$ (see [31]). When $\left(S^{2} \times S^{2}, J\right)$ has two embedded $\mathbb{C P}^{1}$ 's with self-intersection 0 and themselves intersecting at one point, then $\left(S^{2} \times\right.$ 
$\left.S^{2}, J\right) \cong H_{0}$. When $\left(S^{2} \times S^{2}, J\right)$ has an embedded $\mathbb{C P}^{1}$ with self-intersection $-2 k<0$, then $\left(S^{2} \times S^{2}, J\right) \cong H_{2 k}$.

To understand which of these complex structures $J$ can be made compatible with a symplectic form $\omega_{\lambda}$, for some $1 \leq \lambda \in \mathbb{R}$, it is important to note that the compatibility condition implies that the symplectic form evaluates positively on any $J$-holomorphic curve. Hence, for a compatible $J \in X_{\lambda}$, a homology class $(m, n) \in H_{2}\left(S^{2} \times S^{2} ; \mathbb{Z}\right)$ can only be represented by a $J$-holomorphic curve if $\lambda m+n>0$. This rules out embedded curves with self-intersection less than $-2 \ell$, where $\ell \in \mathbb{N}_{0}$ is such that $\ell<\lambda \leq \ell+1$. In particular, the class $(1,-k) \in H_{2}\left(S^{2} \times S^{2} ; \mathbb{Z}\right)$, with self-intersection $-2 k$, can only be represented by a $J$-holomorphic curve for some $J \in X_{\lambda}$ if $\lambda-k>0$.

This turns out to be the only relevant condition. In fact, we have the following theorem.

Theorem 7.2. Given $1 \leq \lambda \in \mathbb{R}$, there is a stratification of $X_{\lambda}$ of the form

$$
X_{\lambda}=V_{0} \sqcup V_{1} \sqcup \cdots \sqcup V_{\ell},
$$

with $\ell \in \mathbb{N}_{0}$ such that $\ell<\lambda \leq \ell+1$ and where:

(i)

$$
\begin{aligned}
V_{k} \equiv\left\{J \in X_{\lambda}:\right. & \left.\left(S^{2} \times S^{2}\right) \cong H_{2 k}\right\} \\
=\left\{J \in X_{\lambda}:\right. & (1,-k) \in H_{2}(M, \mathbb{Z}) \text { is represented } \\
& \text { by a } J \text {-holomorphic sphere }\} .
\end{aligned}
$$

(ii) $V_{0}$ is open and dense in $X_{\lambda}$. For $k \geq 1, V_{k}$ has codimension $4 k-2$ in $X_{\lambda}$.

(iii) $\overline{V_{k}}=V_{k} \sqcup V_{k+1} \sqcup \cdots \sqcup V_{\ell}$.

(iv) For each $k \in \mathbb{N}_{0}$, there is a complex structure $J_{k} \in V_{k}$, unique up to the action of $G_{\lambda}$, for which $g_{\lambda, k} \equiv \omega_{\lambda}\left(\cdot, J_{k} \cdot\right)$ is an extremal Kähler metric.

(v) Denoting by $K_{k}$ the Kähler isometry group of $\left(S^{2} \times S^{2}, \omega_{\lambda}, J_{k}\right)$, we have that

$$
K_{k} \cong \begin{cases}\mathbb{Z} / 2 \ltimes(S O(3) \times S O(3)), & \text { if } k=0 ; \\ S^{1} \times S O(3), & \text { if } k \geq 1 .\end{cases}
$$

(vi) Given $J \in V_{k}$, there exists $\varphi \in \operatorname{Diff}\left(S^{2} \times S^{2}\right)$ such that

$$
\left[\varphi^{*}\left(\omega_{\lambda}\right)\right]=\left[\omega_{\lambda}\right] \in H^{2}\left(S^{2} \times S^{2} ; \mathbb{R}\right) \quad \text { and } \quad \varphi_{*}\left(J_{k}\right)=J
$$


so each stratum $V_{k}$ consists of a unique " $G_{\lambda}^{\mathbb{C}}$ "-orbit, the orbit through $J_{k}$. Moreover, the inclusion

$$
\begin{aligned}
\left(G_{\lambda} / K_{k}\right) & \longrightarrow V_{k}=\mathcal{O}_{J_{k}} \\
{[\psi] } & \longmapsto \psi_{*}\left(J_{k}\right)
\end{aligned}
$$

is a weak homotopy equivalence.

(vii) Each $V_{k}$ has a tubular neighborhood $N V_{k} \subset X_{\lambda}$, with normal slice given by

$$
H^{1}\left(H_{2 k}, \Theta\right) \cong \mathbb{C}^{2 k-1}
$$

where $\Theta=$ sheaf of holomorphic vector fields on $H_{2 k}$.

(viii) For $k \geq 1$, the representation of $K_{k} \cong S^{1} \times S O(3)$ on the normal slice $\mathbb{C}^{2 k-1}$ at $J_{k} \in V_{k}$ is the following: $S^{1}$ acts diagonally and $S O(3)$ acts irreducibly with highest weight $2(k-1)$.

Proof. Points (i), (ii), (iii), (v) and (vii) follow from standard complex geometry and deformation theory applied to complex structures on $S^{2} \times S^{2}$ (see $[15$, I.6]). One needs to check that standard deformation theory can in fact be used here, in the context of compatible complex structures. This, together with points (vi) and (viii), is proved in [7]. Point (iv) is proved in $[12]$.

This theorem shows that the geometric picture suggested by the moment map framework of section 6 is quite accurate for rational ruled surfaces. It implies, by standard equivariant cohomology theory (see [7]), the following corollary.

Corollary 7.3. Given $\ell \in \mathbb{N}_{0}$ and $\left.\left.\lambda \in\right] \ell, \ell+1\right]$, we have that

$$
\begin{gathered}
H_{G_{\lambda}}^{*}\left(X_{\lambda} ; \mathbb{Z}\right) \cong H^{*}(B S O(3) \times B S O(3) ; \mathbb{Z}) \oplus \oplus_{k=1}^{\ell} \Sigma^{4 k-2} \\
H^{*}\left(B S^{1} \times B S O(3) ; \mathbb{Z}\right),
\end{gathered}
$$

where $\cong$ indicates a group isomorphism.

Contractibility of $X_{\lambda}$. Given $\ell \in \mathbb{N}_{0}$ and $\left.\left.1 \leq \lambda \in\right] \ell, \ell+1\right]$, we can combine the stratification of $X_{\lambda}$ described in Theorem 7.2 with the stratification of $\mathcal{J}_{\lambda}$ described in section 2 to obtain a finite family of diagrams, one for each $0 \leq k \leq \ell$, of the form

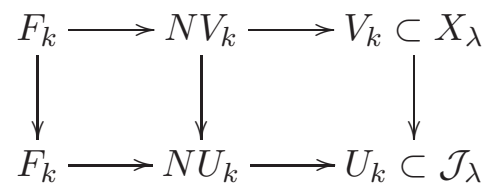

where the vertical arrows are inclusions, the one on the left representing the identity between the fibers of the tubular neighborhoods over $V_{k} \subset U_{k}$. 
These diagrams are $G_{\lambda}$-equivariant in a suitable sense. Given that $\mathcal{J}_{\lambda}$ is contractible and $V_{k}$ is weakly homotopy equivalent to $U_{k}$, one can use this finite family of diagrams to prove the following theorem (see [7]).

Theorem 7.4. Given $1 \leq \lambda \in \mathbb{R}$, the space $X_{\lambda}$ of compatible integrable complex structures on $\left(S^{2} \times S^{2}, \omega_{\lambda}\right)$ is weakly contractible.

As far as we know, these are the first known examples of dimension greater than two where the topology of the space of compatible integrable complex structures is understood.

Cohomology of $B G_{\lambda}$. Theorem 7.4 implies that

$$
H_{G_{\lambda}}^{*}\left(X_{\lambda} ; \mathbb{Z}\right) \cong H^{*}\left(B G_{\lambda} ; \mathbb{Z}\right) .
$$

Combining this isomorphism with Corollary 7.3, we get the following theorem.

Theorem 7.5. Given $\ell \in \mathbb{N}_{0}$ and $\left.\left.\lambda \in\right] \ell, \ell+1\right]$, we have that

$$
\begin{aligned}
H^{*}\left(B G_{\lambda} ; \mathbb{Z}\right) \cong & H^{*}(B S O(3) \times B S O(3) ; \mathbb{Z}) \oplus \oplus_{k=1}^{\ell} \Sigma^{4 k-2} \\
& H^{*}\left(B S^{1} \times B S O(3) ; \mathbb{Z}\right),
\end{aligned}
$$

where $\cong$ indicates a group isomorphism.

\section{Remark 7.6.}

(i) When $1<\lambda \leq 2$, this generalization of Theorem 3.3 is a simple corollary of the work of Anjos and Granja in [8].

(ii) Away from the prime 2, the ring structure of $H^{*}\left(B G_{\lambda}\right)$ is also determined in [7], correcting in particular an incomplete formula for its rational ring structure determined in [2] 


\section{References}

[1] M. Abreu, Topology of symplectomorphism groups of $S^{2} \times S^{2}$, Invent. Math. 131 (1998), 1-23.

[2] M. Abreu and D. McDuff, Topology of symplectomorphism groups of rational ruled surfaces, J. Amer. Math. Soc. 13 (2000), 971-1009.

[3] M. Abreu, Kähler geometry of toric varieties and extremal metrics, Internat. J. Math. 9 (1998), 641-651.

[4] M. Abreu, Kähler geometry of toric manifolds in symplectic coordinates, in "Symplectic and Contact Topology: Interactions and Perspectives" (eds. Y.Eliashberg, B.Khesin and F.Lalonde), Fields Institute Communications 35, American Mathematical Society, 2003, pp. 1-24.

[5] M. Abreu, Kähler metrics on toric orbifolds, J. Differential Geom. 58 (2001), $151-187$.

[6] M. Abreu, G. Granja and N. Kitchloo, Moment maps, symplectomorphism groups and compatible complex structures, J. Symplectic Geom. 3 (2005), 655670 .

[7] M. Abreu, G. Granja and N. Kitchloo, Symplectomorphism groups and compatible complex structures on rational ruled surfaces, preprint (2006), math.SG/0610436.

[8] S. Anjos and G. Granja, Homotopy decomposition of a symplectomorphism group of $S^{2} \times S^{2}$, Topology 43 (2004), 599-618.

[9] M. F. Atiyah, Convexity and commuting Hamiltonians, Bull. London Math. Soc. 14 (1982), 1-15.

[10] A. Besse, "Einstein Manifolds", Springer-Verlag, 1987.

[11] R. Bryant, Bochner-Kähler metrics, J. Amer. Math. Soc. 14 (2001), 623-715.

[12] E. Calabi, Extremal Kähler metrics, in "Seminar on Differential Geometry" (ed. S.T.Yau), Annals of Math. Studies 102, Princeton Univ. Press, 1982, 259-290.

[13] E. Calabi, Extremal Kähler metrics II, in "Differential Geometry and Complex Analysis" (eds. I.Chavel and H.M.Farkas), Springer-Verlag, 1985, 95-114.

[14] E. Calabi and X. X. Chen, The space of Kähler metrics. II, J. Differential Geom. 61 (2002), no. 2, 173-193.

[15] F. Catanese, Moduli of algebraic surfaces, Lecture Notes in Math. 1337, Springer, 1988, 1-83.

[16] T. Delzant, Hamiltoniens périodiques et images convexes de l'application moment, Bull. Soc. Math. France 116 (1988), 315-339.

[17] S. Donaldson, Remarks on gauge theory, complex geometry and 4-manifold topology, in "Fields Medallists' Lectures" (eds. M.F. Atiyah and D. Iagolnitzer), World Scientific, 1997, 384-403.

[18] S. Donaldson, Symmetric spaces, Kähler geometry and Hamiltonian dynamics, in "Northern California Symplectic Geometry Seminar" (eds. Y. Eliashberg et al.), American Mathematical Society, 1999, 13-33.

[19] S. Donaldson, Scalar curvature and projective embeddings I, J. Differential Geom. 59 (2001), 479-522.

[20] S. Donaldson, Scalar curvature and stability of toric varieties, J. Differential Geom. 62 (2002), 289-349.

[21] S. Donaldson, Interior estimates for solutions of Abreu's equation, Collect. Math. 56 (2005), 103-142.

[22] S. Donaldson, Extremal metrics on toric surfaces I, preprint (2006), math.DG/0612120. 
[23] M. Gromov, Pseudo holomorphic curves in symplectic manifolds, Invent. Math. 82 (1985), 307-347.

[24] V. Guillemin, Kähler structures on toric varieties, J. Differential Geometry 40 (1994), 285-309.

[25] V. Guillemin and S. Sternberg, Convexity properties of the moment mapping, Invent. Math. 67 (1982), 491-513.

[26] A. Hwang, On the Calabi energy of extremal Kähler metrics, Internat. J. Math. 6 (1995), 825-830

[27] E. Lerman and S. Tolman, Hamiltonian torus actions on symplectic orbifolds and toric varieties, Trans. Amer. Math. Soc. 349 (1997), 4201-4230.

[28] D. McDuff, Almost complex structures on $S^{2} \times S^{2}$, Duke Math. J. 101 (2000), $135-177$.

[29] D. McDuff and D. Salamon, J-holomorphic Curves and Symplectic Topology, Colloquium Publications, American Mathematical Society, 2004.

[30] J. Moser, On the volume elements on a manifold, Trans. Amer. Math. Soc. 120 (1965), 286-294.

[31] Z. Qin, Complex structures on certain differentiable 4-manifolds, Topology 32 (1993), 551-566.

[32] C. L. Siegel, Symplectic geometry, Academic Press, 1964. 